\title{
Method for Mitigation of NLOS Error based on SVM using Geometry Information and Training data
}

\author{
Jiyan Huang ${ }^{431^{*}}$, Peng Liư ${ }^{2}$, Mingquan $\mathrm{Lu}^{4}$, Baogen $\mathrm{Xu}^{3}$, Zhongchu Rao ${ }^{3}$, Yihe \\ $\mathrm{Wan}^{3}$, Chuan $\mathrm{Lu}^{1}$, and Hongyan Zhang ${ }^{1}$ \\ ${ }^{1}$ Dept. of Electronic Engineering, University of Electronic Science and Technology of China, \\ Chengdu, China. \\ ${ }^{2}$ Space Star Technology Co., Ltd,Beijing, China \\ ${ }^{3}$ Tong Fang Electronic Technology Company Limited, Jiangxi, China \\ ${ }^{4}$ Tsinghua University, BeiJing, China \\ *Jiyan Huang is the corresponding author. \\ huangjiyan@uestc.edu.cn
}

Keywords: Time of arrival, Mobile location, NLOS error, LS-SVM, Kernel function

\begin{abstract}
In this paper, a novel least square support vector machines (LS-SVM) based on geometry information and training data is proposed to improve the performance of mobile localization in the non-line-of-sight (NLOS) environments. Simulations show that the performance of the proposed method is better than the geometry method and the learning method based RBF kernel function in difference channel environments.
\end{abstract}

\section{Introduction}

The location estimation of a mobile station (MS) in a wireless system has gained considerable attention over the past decade, especially since the Federal communication commission (FCC) passed a mandate requiring cellular providers to generate location estimates for Enhance-911 (E-911) services. For TOA-based cellular wireless location systems, NLOS propagation is a kill issue to improve location accuracy [1].

There are several ways to cope with NLOS errors. The first way is using scattering model [2], the difficulty is in obtaining accurate mode. The second way is using constraint condition [3], the problem is that the answer can be unreliablebecause NLOS errors , though reduce, are always present. The third way is the statistical method [4], but the performance is limited by the NLOS error variance.

Because NLOS errors are decided by environments and may change with environments and seasons, the general geometry method can't effectively deal with NLOS errors. So, the training method is developed to restrain NLOS errors. Such as, the training method based on neural network, but the problem is that layers of neural network can't be easily decided and it is easily over fitting. Recently, a kind of new method (RBF kernel LS-SVM method [5]) is proposed to localize MS. The method, using RBF kernel function to learn NLOS errors distribution, can improve the performance of MS localization when NLOS errors are large; but it can't make use of the geometry information, when NLOS errors are small or MS is in the LOS environments, the performance of that method is worse than general geometry method. In this paper, a novel method both using geometry information and training data is proposed to restrain NLOS errors. The method constructs the new kernel function using the geometry information instead of RBF kernel function in [5], and then uses the LS-SVM method to learn NLOS errors distribution. Because of the both using geometry information and training data, the performance of the proposed method is better thanthe geometry method and the training method based RBF kernel function in both LOS environments and NLOS environments. 


\section{LS-SVM location method based on RBF kernel}

Originally, SVM method is introduced within the context statistical learning theory and is used to solve the quadratic program. LS-SVM is reformulation to the standard SVM, which leads to solving linear KKT (Karysh-Kuhn-Tucker) systems. Here, we simply present the basic principle of LS-SVM for function estimation. Given a training data set of $N$ points

$$
S=\left\{\left(\mathrm{x}_{k}, y_{k}\right) \mid K=1,2, \ldots, N\right\} \text { (1) }
$$

With input data $x_{k} \in R^{n}$ and output data $y_{k} \in R$, one considers the following optimization problem in primal weight space. In the weight space, optimal problems can be described as

$$
\min _{w, b, e} J(W, e)=\frac{1}{2} W^{T} W+\frac{1}{2} \gamma \sum_{k=1}^{N} e_{k}^{2}
$$

Subject to

$$
\mathrm{y}_{k}=w^{T} \varphi\left(x_{k}\right)+b+e_{k}, k=1,2, \ldots, N
$$

Where $\varphi(): R^{n} \rightarrow R^{n_{h}}$ is a nonlinear mapping in kernel space, $w \in R^{n_{h}}$, error variable $e_{k} \in R$, and $b$ is a bias, $J$ is a loss function and $\gamma$ is an adjustable constant. The aim of the mapping function in kernel space is picking out features from primal space and mapping training data into a vector of a high dimensional feature space in order to solve the nonlinear regression problem.

According to optimal function (2), the Lagrangian function as:

$$
L(w, b, e, a)=J(w, e)-\sum_{k=1}^{N} a_{k}\left\{W^{T} \varphi\left(x_{k}\right)+b+e_{k}-y_{k}\right\}
$$

Where ${ }^{a_{k}}$ are Lagrange multipliers, also known as support vectors. The optimality of upper function is asfollowing sets of linear equation instead of quadratic program in the traditional SVM.

$$
\left\{\begin{array}{c}
\frac{\partial L}{\partial W}=0 \rightarrow W=\sum_{k=1}^{N} a_{k} \varphi\left(x_{k}\right) \\
\frac{\partial L}{\partial b}=0 \rightarrow \sum_{k=1}^{N} a_{k}=0 \\
\frac{\partial L}{\partial e_{k}}=0 \rightarrow a_{k}=\gamma e_{k} \\
\frac{\partial L}{\partial a_{k}}=0 \rightarrow W^{T} \varphi\left(x_{k}\right)+b+e_{k}-y_{k}=0
\end{array}\right.
$$

Here, $k=1, \ldots, N$. After eliminating variables $(w, e)$, we get matrix equations:

$$
\left[\begin{array}{cc}
0 & 1_{V}^{T} \\
1_{V} & \Omega+\frac{1}{\gamma} I
\end{array}\right]\left[\begin{array}{l}
\mathrm{b} \\
\mathrm{a}
\end{array}\right]=\left[\begin{array}{l}
0 \\
y
\end{array}\right]
$$

Where $y=\left[y_{1}, \ldots, y_{N}\right]^{T}, \quad 1_{v}=[1, \ldots, 1]^{T}, \quad a=\left[a_{1}, \ldots, a_{N}\right], \quad \Omega_{k 1}=\varphi\left(x_{k}\right) \cdot \varphi\left(x_{1}\right)$, $k, 1=1, \ldots, N$ 。

According to Mercer's condition, there is mapping $\varphi$ and kernel function:

Which causes such

$$
\mathrm{K}\left(x_{k}, x_{l}\right)=\left(\varphi\left(x_{k}\right) \cdot \varphi\left(x_{l}\right)\right)
$$

$$
\mathrm{y}(\mathrm{x})=\sum_{\mathrm{k}=1}^{\mathrm{N}} a_{k} K\left(x, x_{k}\right)+b
$$

Where $a, b$ are obtained by solving (6). The RBF kernel is used to localize MS in [5]:

$$
K\left(x_{k}, x_{1}\right)=\exp \left(-\frac{\left|x_{k}-x_{1}\right|^{2}}{2 \sigma^{2}}\right)
$$

There are two parameters to be tuned: the kernel setting $\sigma$ and $\gamma$. 


\section{A new kernel function based on geometry information}

General training method, such as neural network and LS-SVM doesn't use geometry information and can be further improved. In this paper, the geometry information is used to construct a new kernel function and a training method based on new kernel function is proposed to improve the location precision.

Let there be Base stations (BSs) at $\left(B S x_{i}, B S_{y_{i}}\right)$ andmobile station (MS) at ${ }^{(M S x, M S y)}$, the true distance between MS and the $i$ 'th $\mathrm{BS}$ is ${ }^{r_{i}}$, the range measurement equation can be modeled as:

$$
L_{i}=r_{i}+\operatorname{los}_{i}+n \operatorname{los}_{i}=r_{i}+n_{i}(10)
$$

Where, ${ }^{l o s_{i}}$ is measurement noise subjected to Gauss distribution and $n l o s_{i}$ is NLOS error changing with environments. From geometry relation between MS and BSs, we can get:

$$
\begin{aligned}
& \text { Where, } \quad A=\left[\begin{array}{cc}
2\left(B S x_{2}-B S x_{1}\right) & 2\left(B S y_{2}-B S y_{1}\right) \\
\vdots & \vdots \\
2\left(B S x_{M}-B S x_{M-1}\right) & 2\left(B S y_{M}-B S y_{M-1}\right)
\end{array}\right], \quad X=\left[\begin{array}{c}
M S x \\
M S y
\end{array}\right], \\
& B=\left[\begin{array}{c}
r_{1}^{2}-r_{2}^{2}+K_{2}-K_{1} \\
\vdots \\
r_{M-1}^{2}-r_{M}^{2}+K_{M}-K_{M-1}
\end{array}\right], k_{i}=B S x_{i}^{2}+B S y_{i}^{2}, i=1,2, \ldots, M, M \text { is the number of BSs. }
\end{aligned}
$$

Putting (10 into (11), we can get:

$$
\text { where, } B_{1}=\left[\begin{array}{c}
L_{1}^{2}-L_{2}^{2}+K_{2}-K_{1} \\
\vdots \\
L_{M-1}^{2}-L_{M}^{2}+K_{M}-K_{M-1}
\end{array}\right], B_{2}=\left[\begin{array}{c}
A X=B_{1}+B_{2} \\
n_{1}^{2}-n_{2}^{2}-2 L_{1} n_{1}+2 L_{2} n_{2} \\
\vdots \\
n_{M-1}^{2}-n_{M}^{2}-2 L_{M-1} n_{M-1}+2 L_{M} n_{M}
\end{array}\right] .
$$

From (13), we have:

$$
\begin{aligned}
& X=\left[\begin{array}{l}
M S X \\
M S y
\end{array}\right]=\left(A^{T} A\right)^{-1} A^{T} B_{1}+\left(A^{T} A\right)^{-1} A^{T} B_{2} \\
& =C B_{1}+C B_{2}=\left[\begin{array}{l}
\sum_{i=1}^{M-1} C(1, i) B_{1}(i) \\
\sum_{i=1}^{M-1} C(2, i) B_{1}(i)
\end{array}\right]+C B_{2}
\end{aligned}
$$

From (13), the solution of MS position is divided into two parts, geometry determinate part and error compensatory part, so a new kernel function can be constructed by $\left(A^{T} A\right)^{-1} A^{T} B_{1}$ to describe geometry determinate part, then training data is used to learn error distribution and compensate system.

Nonlinear mapping $\varphi(x)$ is constructed according to (5) and (13):

$$
\varphi(x)=\left[\begin{array}{l}
\varphi_{M S X}(x) \\
\varphi_{M S Y}(x)
\end{array}\right]=\left[\begin{array}{l}
\sum_{i=1}^{M-1} C(1, i) B_{1}(i) \\
\sum_{i=1}^{M-1} C(2, i) B_{1}(i)
\end{array}\right]
$$

According to (14), constructing the new kernel function:

$$
K\left(x_{k}, x_{1}\right)=\left[\begin{array}{l}
K_{M S X}\left(x_{k}, x_{1}\right) \\
K_{M S Y}\left(x_{k}, x_{1}\right)
\end{array}\right]=\left[\begin{array}{l}
\left(\varphi_{M S X}\left(x_{k}\right) \cdot \varphi_{M S X}\left(x_{1}\right)\right) \\
\left(\varphi_{M S Y}\left(x_{k}\right) \cdot \varphi_{M S Y}\left(x_{1}\right)\right)
\end{array}\right]
$$


Putting (15) into (8), we can get two group tuning parameters $\left(a_{1}, b_{1}\right)$ and $\left(a_{2}, b_{2}\right)$. The finally solution of MS position is that:

$$
\left[\begin{array}{l}
M S X \\
M S y
\end{array}\right]=\left[\begin{array}{l}
\sum_{\mathrm{k}=1}^{N} a_{k 1} K_{M S x}\left(x, x_{k}\right)+b_{1} \\
\sum_{\mathrm{k}=1}^{N} a_{k 2} K_{M S y}\left(x, x_{k}\right)+b_{2}
\end{array}\right]
$$

Here, $\gamma$ should be chose. Steps of choosing parameter $\gamma$ are that:

1) Choose the scope of parameter $\gamma$.

2) Select the value of $\gamma$ that produced the model that gave the smallest prediction error.

3) Use the optimal parameter to solve MS position (test data is not used for training).

\section{Simulation}

There are seven BS and their locations are at $(0,0), \quad(866,500), \quad(0,1000)$, $(-866,500),(-866,-500),(0,-1000),(866,-500)$, and BS $(0,0)$ is service BS, MS is distributed uniform around the service BS. Here, we defined the position error shown as:

$$
\text { Error }=\sqrt{(M S \hat{x}-M S X)^{2}+(M S \hat{y}-M S y)^{2}}
$$

Where, $\left(M S_{X}, M S_{y}\right)$ is the real position coordinates of MS, $(M S \hat{x}, M S \hat{y})$ is the estimated position coordinates of MS. The proposed algorithm is compared with the geometry method (Least square method) [6] and the training method (RBF-kernel LS-SVM method) [5] in difference channel environments. The number of training data is 50.
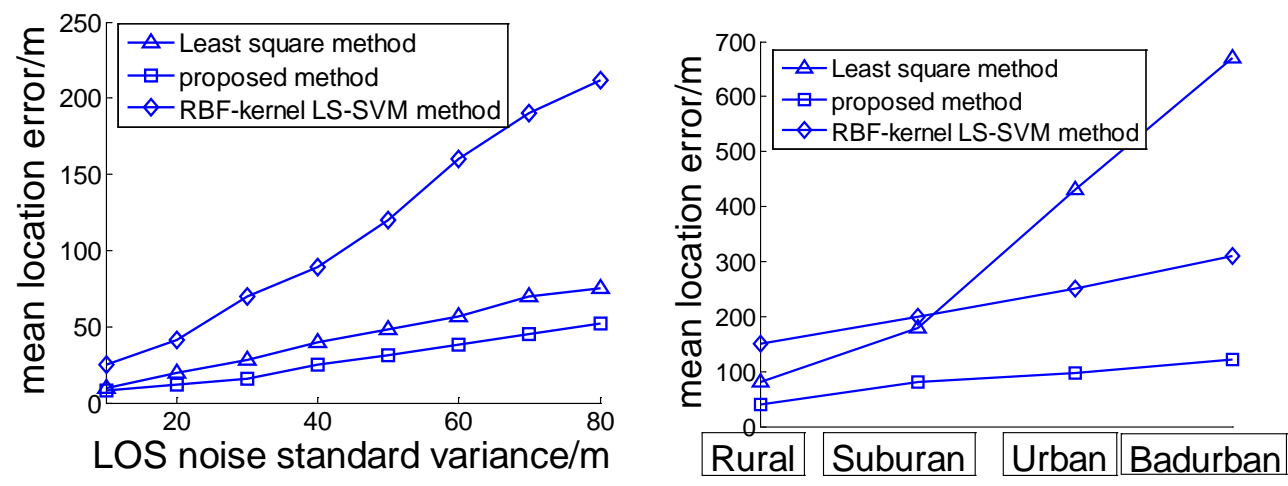

Fig.1 mean location error in LOS environments Fig.2 mean location error in NLOS environments A. performance compares in the LOS environments

For all three algorithms in the LOS environments, the mean location error performance is illustrated and compared in Fig.1. The range measurement noise is subjected Gaussian distribution with zero mean and difference standard variances.From Fig.1, we can conclude that the performance of proposed algorithm is superior to Least square method and RBF-kernel LS-SVM method with difference range measurement noise standard variance.

\section{B. performance compares in the NLOS environments}

For all three algorithms in the NLOS environments, the mean location error performance is illustrated and compared in Fig.2. The range measurement noise is subjected to Gauss distribution with zero mean and standard variance $10 \mathrm{~m}$. NLOS errors are subjected to COST259 channel model [7]. From Fig.2, we can conclude that the performance of proposed algorithm is also superior to Least square method and RBF-kernel LS-SVM method with difference channel model. The RBF-kernel LS-SVM method is superior to Least square method in Urban and Badurban environments, but inferior to Least square method in Rural and Suburan environments. The proposed algorithm both using geometry information and training data can get the best performance in all channel environments. 


\section{Conclusions}

In this paper, a novel LS-SVM localization algorithm both using geometry information and training data is proposed to mitigate NLOS errors in the wireless cellular networks. The method constructs the new kernel function using the geometry information instead of RBF kernel function in [5], and then uses the LS-SVM method to learn NLOS errors distribution. Because of both using geometry information and training data, the performance of the proposed method is better than the geometry method and the learning method based RBF kernel function in LOS environments and NLOS environments.

\section{Acknowledgements}

This work was supported by the Open Research Fund of The Academy of Satellite Application under grant (2014_CXJJ-DH_09), the National Natural Science Foundation of China (61201275), the Fundamental Research Funds for the Central Universities (ZYGX2013J026), China Postdoctoral Science Foundation (2013M540532), and Jiangxi Postdoctoral Research Project (2013KY01). The authors would like to thank the anonymous referees for their suggestions and comments.

\section{References}

[1] J. Caffery, Jr. Wireless Location in CDMA Cellular Radio Systems. Kluwer Academic Publishers, 1999

[2] Wan Q,Yang W L ,Peng Y N. Simplified calculation of mobile location using scatter information. 2004 International Conference on, Communications, Circuits and Systems(ICCCAS 2004),Chengdu, China,2004,1:475-478.

[3] S.Venkatraman, J. Caffery, Jr., and H. R.You, "Location Using LOS range estimation in NLOS environments,” in Proc. IEEE Vehicular Technology Conf., vol. 2, May 2002, pp. 856-860.

[4] Wylie, M.P.; Holtzman, J. The non-line of sight problem in mobile location estimation.Universal Personal Communications, 1996. Record., 1996 5th IEEE International Conference on , Volume: 2 , 29 Sept.-2 Oct. 1996

[5] Sun, G, Guo, W. Robust Mobile Geo-Location Algorithm Based on LS-SVM. Vehicular Technology, IEEE Transactions on Volume 54, Issue 3, May 2005 Page(s):1037 - 1041

[6] J. Caffery, “A new approach to the geometry of TOA location," in Proc.IEEE Vehicular Technology Conf. (VTC’00), 2000, pp. 1943-1949.

[7] Asplind etal. A Channel Model for Positioning, Cost259TD(98)20. Ericsson Corp. Bern, Switzerland,1998 